\title{
命HAD
}

DOI: http://doi.org/10.22585/hospdomic.v3i4.82

\section{Eficacia y seguridad de la Naltrexona en domicilio para el manejo del prurito resistente en paciente oncológico terminal}

Efficacy and safety of Naltrexone at home to the management of resistant pruritus in terminal cancer patient

Aarón Gutiérrez Pastor', María Balboa Alonso², Pablo Tordera Higón'

1. Hospital San Juan de Alicante. Unidad de Hospitalización a Domicilio. Sant Joan d'Alacant, España.

2. Hospital de San Juan de Alicante. Unidad de Urgencias. Sant Joan d'Alacant. España.

Correspondencia/Correspondence

Aarón Gutiérrez Pastor

Recibido/Received

05.07.2019

Aceptado/Accepted

01.09 .2019
Conflicto de Intereses/Competing interest Los autores y autora del trabajo declaran no tener ningún conflicto de interés. 


\section{RESUMEN}

El prurito es una sensación desagradable que supone un gran impacto en la calidad de vida del paciente terminal. Su etiopatogenia es compleja y poco conocida. Una de las teorías es el incremento del tono opioide cerebral, por lo que uno de los tratamientos es la utilización de un antagonista opioide para su control.

Presentamos el caso de un paciente ingresado a cargo de la Unidad de Hospitalización a Domicilio que presentaba prurito intenso a pesar de tratamiento pautado. Se inició tratamiento con Naltrexona por vía oral, presentando mejoría de la sintomatología con resolución del cuadro.

Palabras clave: Prurito; Antagonista opioide; Naltrexona; Servicios de Atención a Domicilio Provisto por Hospital.

\section{ABSTRACT}

Pruritus is an unpleasant sensation that has a great impact on the quality of life of the terminal patient. Its etiopathogenesis is complex and little known. One of the theories is the effect of the cerebral opioid tone, the treatment of an opioid antagonist for its control.

We present the case of a patient admitted to a load of the Hospitalization and Domicile Unit, which presented an intense pruritus despite the prescribed treatment. Treatment with Naltrexone was initiated orally, presenting symptom improvement with resolution of the symptoms.

Keywords: Pruritus; Opioid Antagonist; Naltrexone; Home Care Services, Hospital-Based. 


\section{INTRODUCCIÓN}

El prurito se define como una sensación desagradable que desencadena la necesidad de rascarse (1). Afecta frecuentemente a las palmas de las manos y las plantas de los pies. La etiopatogenia del prurito es compleja y poco conocida al intervenir diversos mediadores químicos, entre ellos, los opioides endógenos.

Entre las causas, se encuentran diferentes patologías de la piel, tumores primarios o metastásicos, síndromes paraneoplásicos, colestasis, insuficiencia renal avanzada, y el inducido por fárma$\cos ($ tabla 1).

\begin{tabular}{|l|l|}
\hline \multicolumn{2}{|l|}{ Tabla 1. Fármacos que pueden producir prurito (11) } \\
\hline Fármacos que inducen & $\begin{array}{l}\text { Antiarrítmicos: amiodarona } \\
\text { Anticonceptivos orales: estrógenos, progestágenos } \\
\text { Anticonvulsivantes: fenitoína } \\
\text { Anthipipertensivos: captopril } \\
\text { Anabolizantes hormonales: metiltestosterona } \\
\text { Hipoglucemiantes orales: clorpropamida, tolbutamida } \\
\text { Psicóármacos: clorpromacina, fenotiacinas } \\
\text { Quimioterápicos: cloramfenicol, sulfamidas, eritromicina, estolato }\end{array}$ \\
\hline $\begin{array}{l}\text { Fármacos que provocan } \\
\text { liberación de Histamina }\end{array}$ & $\begin{array}{l}\text { Alcaloides: atropina, codeína, papaverina, morfina } \\
\text { Antibióticos: clortetraciclina, neomicina, polimixina B } \\
\text { Antihipertensivos: hidralazina, reserpina } \\
\text { Salicilatos: ácido acetilsalicílico }\end{array}$ \\
\hline Otros & \\
\hline & $\begin{array}{l}\text { Quinidina, cloroquina, vitaminas del grupo B, interferón alfa, } \\
\text { barbitúricos }\end{array}$ \\
\hline
\end{tabular}

Es uno de los síntomas de difícil control en cuidados paliativos, teniendo un gran impacto en la calidad de vida del paciente. Puede alterar el normal desarrollo cognitivo, así como producir insomnio, depresión, ansiedad, lesiones de rascado e incluso culminar en ideas suicidas (2).

En la valoración clínica del prurito, se ha de hacer un examen minucioso de la piel, la cual resulta generalmente normal, o presentar lesiones por rascado. Además, se han de utilizar escalas subjetivas. Entre las más utilizadas se encuentra la escala visual analógica (EVA), que determina la intensidad del prurito en una escala de 0 a 10 puntos, siendo 10 el peor prurito imaginable (3).

El tratamiento del prurito se basa, en primer lugar, en prevenir el contacto con agentes que puedan ocasionarlo, tales como alérgenos cutáneos o farmacológicos así como agentes irritantes de 
la piel. También se ha de evitar la sequedad mediante el empleo de baños de agua tibia o cremas hidratantes o lubricantes (4).

Posteriormente, se ha de centrar en la etiología. El prurito colestásico es reversible cuando la colestasis es obstructiva extrahepática o secundaria a fármacos, pudiendo actuar sobre estas causas para eliminarlo. Sin embargo, la mayoría de procesos asociados a colestasis son crónicos e incurables, por lo que el tratamiento del prurito en estos casos puede basarse en diversos fármacos: Resinas de intercambio iónico no reabsorbibles como Colestiaramina, Colestipol y Colesevalan; Inductores del metabolismo hepático como Fenobarbital y Rifampicina; Antagonistas serotoninérgicos como Paroxetina, Mirtazapina y Ondansetrón; Andrógenos como Metilestosterona o Danazol y, finalmente, los Antagonistas de opioides como la Naloxona y Naltrexona (5) (tabla 2).

Tabla 2. Fármacos utilizados en el tratamiento del prurito (12)

\begin{tabular}{|c|c|c|}
\hline Fármaco & Dosis & Efectos secundarios \\
\hline Colestiramina & $\begin{array}{l}4 \mathrm{~g} \text { v.o. antes y después de desayuno. } \\
\text { Incrementar } 3 \mathrm{~g} \text { en otra comida. } \\
\text { No exceder } 16 \mathrm{~g} / \text { día }\end{array}$ & $\begin{array}{l}\text { Estreñimiento, meteorismo, malabsorción, } \\
\text { coagulopatía }\end{array}$ \\
\hline Naltrexona & $\begin{array}{l}25 \mathrm{mg} / \text { día v.o. el primer día seguido de } \\
50 \mathrm{mg} / \text { día v.o. }\end{array}$ & $\begin{array}{l}\text { Síndrome de abstinencia a opiáceos like. } \\
\text { Hepatotoxicidad }\end{array}$ \\
\hline Naloxona & $\begin{array}{l}0,2 \mathrm{mg} / \mathrm{kg} / \mathrm{min} \text { iv. En infusión continua } \\
\text { precedido de bolus de } 0,4 \mathrm{mg} \text { iv. inicial }\end{array}$ & Síndrome de abstinencia a opiáceos like. \\
\hline Sertralina & 75mg/día v.o. & $\begin{array}{l}\text { Trastornos gastrointestinales, anorexia, } \\
\text { cefalea, sudoración, anorgasmia, Hipertensión } \\
\text { intracraneal, Síndrome serotoninérgico }\end{array}$ \\
\hline Ondansetron & 4-8mg/8h v.o. & $\begin{array}{l}\text { Cefalea, estreñimiento, diarrea, elevación } \\
\text { transaminasas }\end{array}$ \\
\hline Gabapentina & $\begin{array}{l}\text { Iniciar 300mg/día. Ajustar según } \\
\text { respuesta. Dosis mantenimiento: 900- } \\
\text { 3600mg/día repartido en } 3 \text { tomas }\end{array}$ & $\begin{array}{l}\text { Somnolencia, aumento de peso, ataxia, } \\
\text { cefalea, leucopenia }\end{array}$ \\
\hline Butarfanol & 1-2mg/día en spray nasal & Adicción \\
\hline Rifampicina & 300-450mg/día ó 10mg/kg/día & $\begin{array}{l}\text { Hepatotoxicidad, náuseas, vómitos, diarrea, } \\
\text { miopatía, leucopenia, eosinofilia }\end{array}$ \\
\hline
\end{tabular}


Se expone el caso de un paciente con prurito severo, manejado con múltiples tratamiento sin mejoría del cuadro. Se propone manejo con antagonista opioide en dosis ascendente.

\section{DESARROLLO DE LA EXPERIENCIA}

Se presentan el caso clínico de un varón de 79 años de edad con antecedentes de Diabetes Mellitus, Hipertensión arterial, Insuficiencia renal crónica, Demencia por cuerpos de Lewy. Es dependiente para las actividades básicas de la vida diaria, con un resultado en la escala de valoración funcional de Karnofsky de 40. Vive con su mujer en su domicilio particular. Es remitido desde Oncología a la Unidad de Hospitalización a Domicilio de San Juan de Alicante tras diagnóstico hace 6 meses de un Hepatocarcinoma en situación paliativa, al no ser candidato a tratamiento oncológico por presentar bajo nivel en la Escala de Palliative Performance Status.

Analíticamente, presenta hemoglobina de 9g/dl, GOT 48, GPT 53, GGT 45, Fosfatasa alcalina 87, Bilirrubina de 1,7mg/dl e Índice de Quick del 25\%.

Desde el diagnóstico, el paciente ha presentado un deterioro clínico progresivo, con fallo hepático, lo que ha ocasionado episodios de encefalopatía, revertidos con Levomepromazina y Enemas de Lactulosa. Además, ha requerido de transfusiones periódicas y tratamiento con Ácido Tranexámico por anemia de procesos crónicos por coagulopatía agudizada tras episodios de rectorragias.

Actualmente, presenta un prurito de difícil control a pesar de tratamiento con Ácido Ursodesoxicólico y Colestiramina, lo que le genera gran inquietud, molestias e importantes lesiones dérmicas de rascado (figuras 1 y 2 ).

Figura 1. Lesiones de rascado a nivel de Miembro superior derecho

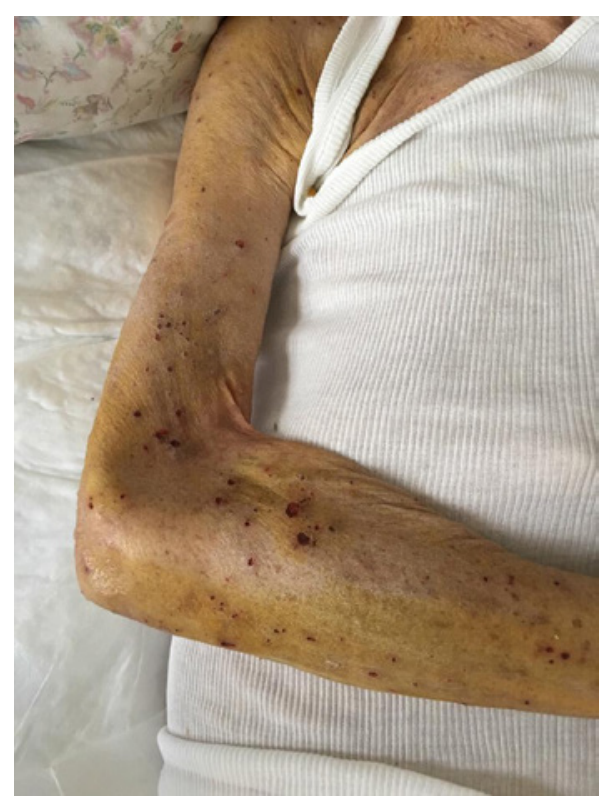

Figura 2. Lesiones de rascado a nivel de ambos miembros inferiores

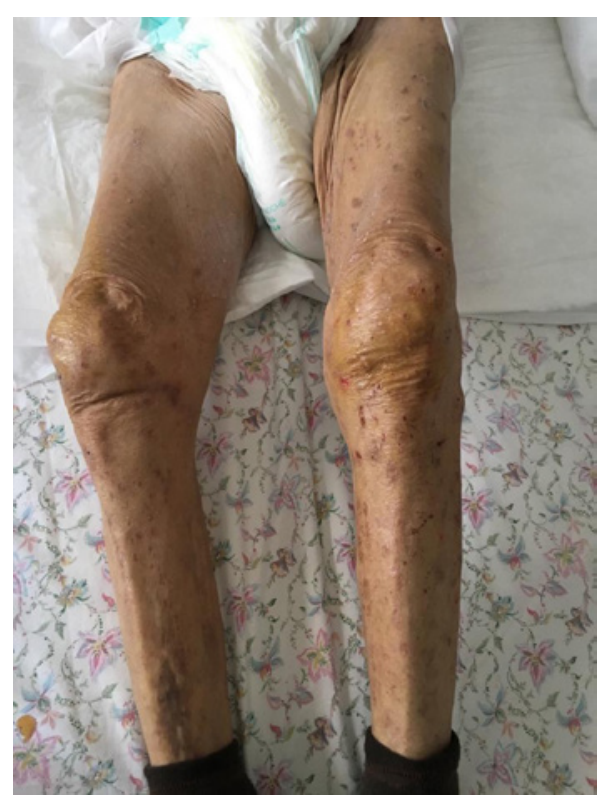


Ante la clínica de la paciente se inició tratamiento con Hidroxizina cada 8 horas; Loratadina $10 \mathrm{mg}$ cada 12 horas; Dexametasona en dosis de $8 \mathrm{mg}$ por la mañana y $4 \mathrm{mg}$ al mediodía e, incluso, Gabapentina 300mg cada 8 horas. Todo ello sin mejoría.

Tras ello, se decide probar con un antagonista opioide endógeno, la Naltrexona, a dosis de $25 \mathrm{mg}$ cada 24 horas durante 7 días y, posteriormente, aumentar a 50mg cada 24 horas. Después de 15 días de tratamiento, se observa una mejoría del cuadro clínico, con reducción de la sensación de prurito y una disminución de la escala visual de prurito. En nuestro caso, el paciente pasó de un resultado de 8/10 en la escala visual analógica de prurito a uno de 3/10 durante las dos semanas de tratamiento, mejorando su calidad de vida y, permitiendo la resolución de sus lesiones de rascado.

\section{CONCLUSIONES}

El prurito es una sensación desagradable que supone un gran impacto en la calidad de vida del paciente terminal. Su etiopatogenia es compleja y poco conocida.

Una de las teorías es el incremento del tono opioide cerebral como parte del componente fisiopatológico en el prurito secundario a trastornos hepáticos, ya sea asociado o no a colestasis. Esta hipótesis está fundamentada en tres razones $(6,7)$ :

- Efecto pruritógenico secundario al incremento del tono opioide

- Incremento en el agonismo opioide cerebral

- Disminución del prurito con la utilización de un antagonista opioide

Estos hechos, se han visto demostrados en diversos estudios controlados con un antagonista opioide, ya sea con tratamiento intravenoso con Naloxona, o tratamiento oral con Naltrexona o Nalmefene $(8,9)$.

Sin embargo, se ha de tener en cuenta que este tratamiento puede desencadenar un respuesta similar a un "síndrome de abstinencia". Debido a ello, es importante iniciar el tratamiento a dosis bajas, aumentando gradualmente hasta obtener la dosis necesaria para obtener efectos terapéuticos (10).

En el caso de nuestro paciente, se ha observado una eficacia del tratamiento por Naltrexona con mejoría de la clínica. Además, no ha presentado efectos secundarios. Debido a ello, concluimos que, la Naltrexona puede ser un fármaco útil y práctico para proporcionar una terapia de control de prurito en aquellos pacientes en situación terminal cuyo tratamiento previo no haya resultado eficaz (figura 3). Sin embargo para la generalización de ésta sería necesario estudios más sólidos y estandarizados que prueben esta hipótesis. 
Figura 3. Algoritmo de tratamiento en prurito colestásico (3)

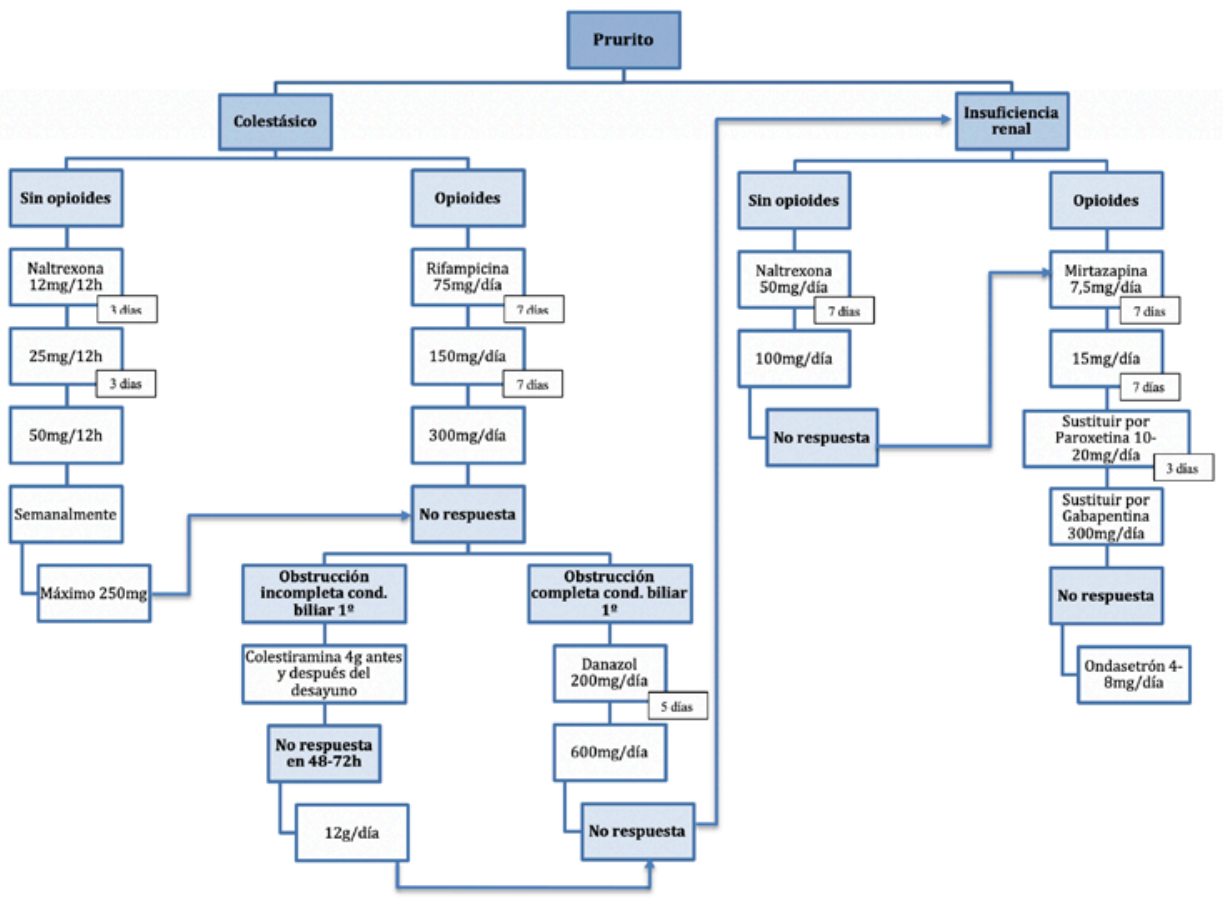

\section{BIBLIOGRAFÍA}

1. Terán A, Fabrega E, Pons-Romero F. Prurito asociado a colestasis. Gastroenterol Hepatol. 2010;33(4):313-22. DOI: 10.1016/j.gastrohep.2009.07.006; PMID: 19836105

2. Olmos O. Prurito generalizado primario. En: Sociedad Española de Medicina de familia y Comunitaria (semFYC). Guía de actuación en Atención Primaria. $4^{a}$ edición. Barcelona, España: semFYC ediciones; 2011. págs. 870-3.

3. Balboa Alonso M, López Bas R. Prurito resistente en paciente en situación paliativa-terminal (Caso clínico 16. UrgePaliar 2018) [monografía en Internet]. Barcelona, España: semFYC ediciones; 2018 [consultada 25 ago 2019]. Disponible en: http://bit.ly/2NG5VVh

4. Fernández Casado A, Segura Tigell S, Pujol Vallverdú RM. Protocolo terapéutico del prurito de la colestasis [monografía en Internet]. Barcelona, España: Servicio de Dermatología del Hospital del Mar; 2007 [consultada 25 ago 2019]. Disponible en: http://bit.ly/2PfZQY8

5. Benítez-Rosario MA, González Guillermo T, editores. Tratamientos protocolizados en cuidados paliativos. Madrid, España: Ediciones YOU \& US; 2010. 
6. Jones EA, Neuberger J. Bergasa NV. Opiate antagonist therapy for the pruritus of cholestasis: The Avoidance of opioide withdrawal-like reactions. Q J Med (QJM). 2002;95(8):547-52. DOI: 10.1093/qjmed/95.8.547; PMID: 12145394

7. Nabal M, Guanter L. Manejo terapéutico de los síntomas difíciles en cuidados paliativos. Med Pal. 2002;9(2):96-101.

8. Mansour-Ghanaei F, Taheri A, Froutan H, Ghofrani H, Nasiri-Toosi M, Bagherzadeh AH, et al. Effect of oral naltrexone on pruritus in cholestatic patients. World J Gastroenterol. 2006;12(7):1125-8.

9. Terg R, Coronel E, Sordá J, Muñoz AE, Findor J. Efficacy and safety of oral naltrexone treatment for pruritus of cholestasis, a crossover, double blind, placebo-controlled study. J Hepatol 2002;37(6):717-22. DOI: 10.1016/s0168-8278(02)00318-5; PMID: 12445410

10. Aristizábal Linares JP, Andrade Almario L. Eficacia de la naltrexona en el tratamiento de prurito severo. Rev Col Anest. 2011;39(2):251-57. DOI: 10.5554/rca.v39i2.91

11. Bóveda E, Díez B, Fernández P, Hidalgo E, Salinas E. Protocolos de atención farmacéutica: prurito. Farmacia profesional. 2003;17(4):68-76.

12. Fernández Casado A, Segura Tigell S, Pujol Vallverdú RM. Protocolo terapéutico del prurito de la colestasis. Servicio de Dermatología. Hospital del Mar. 2007. 